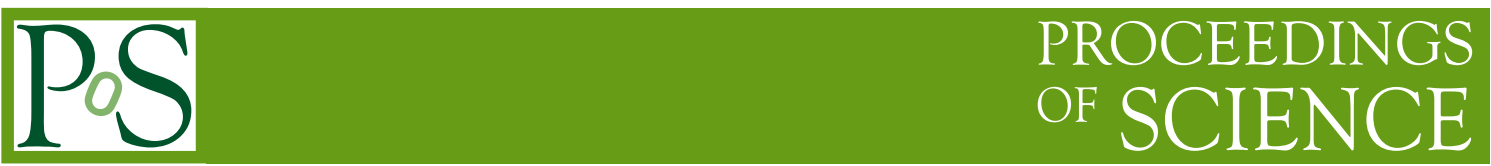

\title{
Twin SMBH candidates in the BCG of RBS 797
}

\author{
Myriam Gitti* \\ Dipartimento di Fisica e Astronomia - Università di Bologna \\ \& INAF - Istituto di Radioastronomia Bologna \\ E-mail: myriam.gittieunibo.it
}

\section{Marcello Giroletti}

INAF - Istituto di Radioastronomia Bologna

E-mail: giroletti@ira.inaf.it

\section{Gabriele Giovannini}

Dipartimento di Fisica e Astronomia - Università di Bologna \& INAF - Istituto di Radioastronomia Bologna

E-mail: ggiovann@ira.inaf.it

\section{Luigina Feretti}

INAF - Istituto di Radioastronomia Bologna

E-mail: lferetti@ira.inaf.it

\section{Elisabetta Liuzzo}

INAF - Istituto di Radioastronomia Bologna

E-mail: liuzzo@ira.inaf.it

The radio-loud BCG at the center of the cool core cluster RBS 797 is known to exhibit a misalignment of its $5 \mathrm{GHz}$ radio emission observed at different VLA resolutions, with the innermost kpc-scale jets being almost orthogonal to the radio lobes which extends for tens of kpc filling the $\mathrm{X}$-ray cavities seen by Chandra. The different radio directions may be caused by rapid jet reorientation due to interaction with a secondary supermassive black hole (SMBH), or to the presence of two AGN, probably in a merging phase, which are emitting radio jets in different directions. We present the results of new $5 \mathrm{GHz}$ observations performed with the EVN in May 2013. In particular, we detected two compact radio components, with a projected separation of $77 \mathrm{pc}$. We discuss two possible scenarios for the origin and nature of the EVN double source, showing that both interpretations are consistent with the presence of a SMBH binary system in the BCG of RBS 797.

12th European VLBI Network Symposium and Users Meeting - EVN 2014,

7-10 October 2014

Cagliari, Italy

\footnotetext{
*Speaker.
} 


\section{Introduction}

The production and coalescence of supermassive black hole (SMBH) binary systems seem to be a natural consequence of galaxy mergers during the formation of cosmological structures (e.g., Begelman et al. 1980). However, observational cases where both SMBHs in a merging system are accreting as active galactic nuclei (AGN) are rare, and there have only been a few confirmed kpc-scale binary AGN (e.g., Colpi \& Dotti 2011, and references therein). The detection of spatiallyresolved dual compact X-ray or radio sources in an active galaxy provides the most unambiguous evidence that a system hosts a SMBH pair. On the other hand, some observational properties of AGN are thought to be indirect evidence of the presence of SMBH binaries, e.g., the interruption and restarting of jet activities in double-double radio galaxies (e.g., Liu et al. 2003) and the rapid jet reorientation in X-shaped radio sources due to the spin-flip of a primary, jet-ejecting SMBH during the interaction with a secondary SMBH in a close system (e.g., Merritt \& Ekers 2002). An understanding of how SMBH binaries form and coalesce is important for the comprehension of the AGN dynamics as well as galaxy formation in general.

\section{The radio properties of RBS 797's BCG}

\section{- On kpc-scale (VLA)}

The radio emission from the brightest cluster galaxy (BCG) in RBS 797 ( $\mathrm{z}=0.35$ ) observed at VLA resolutions shows different orientations of the radio jets and lobes with scale (Gitti et al. 2006). In particular, the kpc-scale radio jets oriented to the north-south (N-S) direction are almost orthogonal to the axis of the diffuse radio emission filling the X-ray cavities on tenkpc scale (Figure 1, left panel). On the other hand, from a recent re-analysis of archival VLA data at $4.8 \mathrm{GHz}$, we find strong evidence of the presence of kpc-scale jets emanating also to the east-west (E-W) direction (Figure 1, middle panel), indicating that the radio-emitting plasma on the E-W direction is still being freshly injected. The two outbursts could thus be almost contemporaneous, suggesting the presence of two active SMBHs whose radio nuclei are unresolved at VLA resolution (Gitti et al. 2013).

\section{- On pc-scale (EVN)}

Since no information on the pc-scale radio properties are available in literature, we performed an explorative program to assess the detectability of the BCG in RBS 797 at VLBI resolution and to study its nuclear region. In particular, on 3 May 2013 we conducted a test observation (PI: M. Gitti) in the $6 \mathrm{~cm}$ e-VLBI run with a subset of the European VLBI Network (EVN). The total time spent on the target was about 1 hour. As it appears evident from the $5 \mathrm{GHz}$ EVN map (Figure 1, right panel), we clearly detected two compact components. The results of the visibility model fit with two Gaussian components, shown in Table 1, indicate that both components are compact and smaller than the observing beam (Gitti et al. 2013).

\section{Possible scenarios}

A definite test to unveil the nature of this source can only be carried out by analyzing deeper images with angular resolution in between VLA and VLBI images, and/or by investigating the 

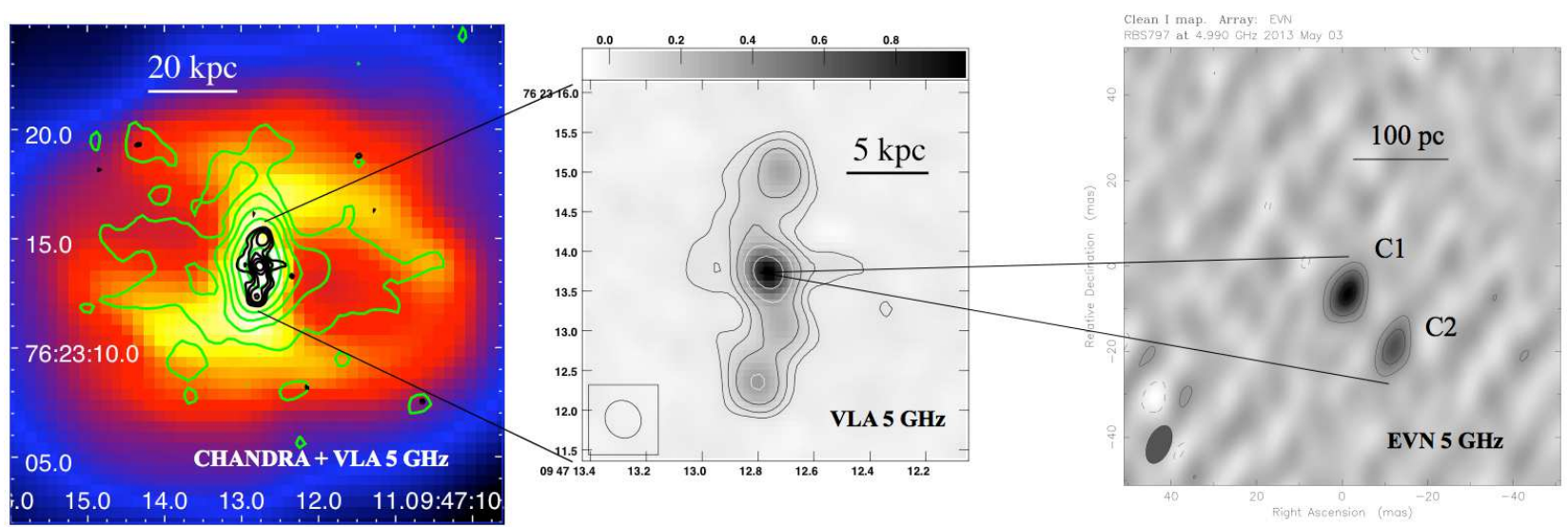

Figure 1: Left panel: The $5 \mathrm{GHz}$ VLA contours of RBS 797, imaged at different resolutions, are overlaid onto the Chandra image of the central region of the cluster. Green contours: $5 \mathrm{GHz}$ VLA map at $1^{\prime \prime} .4 \times 1^{\prime \prime} .3$ resolution; the r.m.s. noise is $0.01 \mathrm{mJy}^{\text {beam }}{ }^{-1}$ and the contour levels start at $3 \sigma$, increasing by a factor 2 . Black contours: see caption middle panel. Middle panel : $5 \mathrm{GHz}$ VLA map at $0^{\prime \prime} .5 \times 0^{\prime \prime} .4$ resolution (the beam is shown in the lower-left corner). The r.m.s. noise is $0.01 \mathrm{mJy}^{\mathrm{beam}}{ }^{-1}$ and the contour levels start at $0.04 \mathrm{mJy}_{\text {beam }}{ }^{-1}$, increasing by a factor 2. Right panel : $5 \mathrm{GHz}$ EVN map of the BCG in RBS 797 at a resolution of $9.4 \times 5.3 \mathrm{mas}^{2}$ in P.A. $24^{\circ}$ (the beam is shown to the lower-left). The r.m.s. noise is $36 \mu \mathrm{Jy}$ beam $^{-1}$ and the peak flux density is $0.53 \mathrm{mJy}_{\text {beam }}{ }^{-1}$. The contours levels start at $3 \sigma$ and increase by a factor 2. The three panels have been adapted from Gitti et al. (2013).

spectral index properties of the different components. We have recently obtained deep, multifrequency follow-up observations of the BCG in RBS 797, which were performed with the VLBA and EVN+eMERLIN in 2014. The data analysis is currently underway and will be presented in a forthcoming paper. Hopefully, the new data will allow us to reach a definite conclusion about the complex origin of the VLBI double source, in particular discriminating between the two following scenarios:

\section{Two nuclei in a binary system}

The scenario that the double source is a close pair of active SMBHs is favored by the compactness of the two VLBI components (Figure 1, right panel) and by the large-scale properties of the radio source (Figure 1, left and middle panels). In this scenario we expect to measure a flat spectrum for both components $\mathrm{C} 1$ and $\mathrm{C} 2$. Each component may also show its own jet (or jet-counterjet pair) emerging from its center. The eMERLIN data will fill in the gap between the pc and the kpc scale, to reveal if both the N-S and E-W channels are currently active, as suggested by our re-analysis of the VLA data (Figure 1, middle panel).

\section{Core-jet structure}

Alternatively, the two components may be the core and a knot of its jet, and the emission from the underlying jet flow connecting the two components may not be visible due to the limited sensitivity of our short observation. The component $\mathrm{C} 1$ is the most likely main core candidate, because of its more compact size and the higher flux density. In the core-jet scenario, the pc-scale jet flow (Figure 1, right panel) would not be aligned with any of the directions seen at kpc-scale in the VLA images (Figure 1, left and middle panels), so the eMERLIN data will be of great importance to reveal where the jet P.A. change occurs. 
Table 1: Gaussian Model Components. Col. (1): Gaussian component as labeled in Figure 1, right panel. Col. (2): Flux density. Cols. (3)-(4): Polar coordinates of the center of the component relative to the position RA: $09^{\mathrm{h}} 47^{\mathrm{m}} 12^{\mathrm{s}} .760$, Dec: $+76^{\circ} 23^{\prime} 13^{\prime \prime} .733$. Cols. (5)-(6): Major axis. Col. (7): Axial ratio. Col. (8): Component orientation. All angles are measured from north to east.

\begin{tabular}{lccccccc}
\hline \hline Comp. & $\begin{array}{c}\text { Flux } \\
(\mathrm{mJy})\end{array}$ & $\begin{array}{c}\mathrm{r} \\
(\mathrm{mas})\end{array}$ & $\begin{array}{c}\theta \\
(\mathrm{deg})\end{array}$ & $\begin{array}{c}\mathrm{a} \\
(\mathrm{mas})\end{array}$ & $\begin{array}{c}\mathrm{a} \\
(\mathrm{pc})\end{array}$ & $\mathrm{b} / \mathrm{a}$ & $\begin{array}{c}\Phi \\
(\mathrm{deg})\end{array}$ \\
\hline & & & & & & & \\
C1 & 0.606 & 0.43 & 29.4 & 2.96 & 14.2 & 1.00 & 58.1 \\
C2 & 0.540 & 16.1 & -139.2 & 7.44 & 35.7 & 0.59 & -1.4 \\
\hline
\end{tabular}

Both interpretations are consistent with the presence of a SMBH binary system. In the scenario (1) the two SMBHs would be both active, detected as two radio nuclei. In the scenario (2) only the primary SMBH would be active (showing a core-jet structure), whereas the secondary SMBH which is likely causing the spin-flip of the primary would remain undetected.

\section{References}

Begelman, M. C., Blandford, R. D., \& Rees, M. J. 1980, Nature, 287, 307

Colpi, M. \& Dotti, M. 2011, Advanced Science Letters, 4, 181

Gitti, M., Feretti, L., \& Schindler, S. 2006, A\&A, 448, 853

Gitti, M., Giroletti, M., Giovannini, G., Feretti, L., \& Liuzzo, E. 2013, A\&A, 557, L14

Liu, F. K., Wu, X.-B., \& Cao, S. L. 2003, MNRAS, 340, 411

Merritt, D. \& Ekers, R. D. 2002, Science, 297, 1310 